\title{
Spontaneous Giant Retinal Pigment Epithelium Rip with Exudative Retinal Detachment-An Unusual Presentation
}

\author{
Vinit J Shah ${ }^{1 *}$, Syed Mohideen Abdul Khadar ${ }^{1}$, Syed Saifuddin Adeel ${ }^{1}$, Shobita Nair ${ }^{1}$ \\ ${ }^{1}$ Department of Retina and Vitreous, Aravind Eye Hospital, Tirunelveli. Tamilnadu, India \\ *Corresponding Author: Vinit J Shah, Department of Retina and Vitreous, Aravind Eye Hospital, Tirunelveli. \\ Tamilnadu, India; Email: drvjshah@gmail.com
}

Received Date: 17-06-2021; Accepted Date: 08-07-2021; Published Date: 15-07-2021

Copyright $^{\oplus} 2021$ by Shah VJ, et al. All rights reserved. This is an open access article distributed under the terms of the Creative Commons Attribution License, which permits unrestricted use, distribution, and reproduction in any medium, provided the original author and source are credited.

\begin{abstract}
Retinal Pigment Epithelial (RPE) rip or tears are known to occur as a natural result in the course of a retinal Pigment Epithelial Detachment (PED) in patients with neovascular Age-Related Macular Degeneration (AMD) or response to intravitreal anti-VEGF or laser for Wet AMD. After endophthalmitis, the most dreaded complications post Anti-VEGF injection is RPE tears leading to detrimental visual loss.

Here, we report a case of a 57-year-old diabetic male patient who developed exudative retinal detachment in the left eye who underwent inguinal hernia surgery 20 days back. Uveitic workup done for exudative detachment was normal. FFA showed transmitted choroidal hyper fluorescence in the early phase suggestive of RPE rip and in late phase dye pooling in bullous detachment. The patient underwent Retinal detachment surgery with unexpected post-operative re-detachment noted at 1 week. This report highlights giant RPE rip as an uncommon cause for extensive exudative detachment, its evaluation, and difficult surgical management.
\end{abstract}

\section{Keywords}

Giant RPE Rip; Exudative Retinal Detachment; Scleral Buckling; Pars plana Vitrectomy; Choroidal Hyperpermeability 


\section{Introduction}

Retinal Pigment Epithelial (RPE) rips occur rarely and are usually associated with Pigment Epithelium Detachments (PED), secondary to Choroidal Neovascular Membrane (CNVM) and polypoidal choroidal vasculopathy which classically involves macula with rapid visual loss [1]. Mid peripheral and peripheral RPE tears are uncommon and tend to be much larger. RPE tears outside the macula are seen in patients with rhegmatogenous retinal detachment, Central Serous Retinopathy (CSR), choroidal tumor, blunt trauma, panuveitis, and after glaucoma surgery [2].

Casswell, et al., reported that spontaneous tear rate of vascular PED is 10\% [3]. To the best of our knowledge spontaneous giant RPE rip causing extensive exudative retinal detachment has seldom been reported. Herein we report a case of extensive exudative retinal detachment caused by spontaneous RPE rip with no other retinochoroidal lesions.

\section{Case Report}

A 57-Year-old male patient presented to our hospital with left eye sudden painless loss of superior field of vision for 3 days. Best-corrected visual acuity in the right eye was $6 / 6$ and the left eye was 6/60. The patient was a known diabetic for 6 years on regular treatment and gave a history of undergoing Inguinal Hernia surgery 20 days back.

Both eyes anterior segment examination was normal except for immature senile cataract. Fundus examination of the right eye showed mild NPDR changes and the left eye showed Bullous exudative retinal detachment (Fig. 1) with shifting fluid which was confirmed on Bscan there was no evidence of any choroidal mass. The uveitic workup of the patient was within normal limits. FFA was done which showed window defect in the early phase in the superotemporal quadrant suggestive of RPE rip with late-phase showing leakage and pooling of dye in bullous retinal detachment (Fig. 2). His vision further deteriorated to 3/60 within one week.

A diagnosis of left eye exudative retinal detachment following RPE rip was made and the patient underwent scleral buckling with vitrectomy and silicone oil injection surgery. Intraoperatively no retinal breaks where found and a drainage retinotomy was done in inferotemporal quadrant to drain the fluid and was lasered. The RPE rip extended during induction of posterior vitreous detachment. On $1^{\text {st }}$ postoperative day vision was $6 / 60$ and fundus showed attached retina under silicone oil (Fig. 3). On one-week follow-up fundus showed shallow retinal detachment in superior quadrant (Fig. 3) for which observation with strict prone position was advised, and at subsequent visit, the retina settled without any intervention. On follow-up visit at 6 months patient's vision in LE improved to 6/36, the retina was attached under silicone oil (Fig. 3) and he underwent silicone oil removal. 

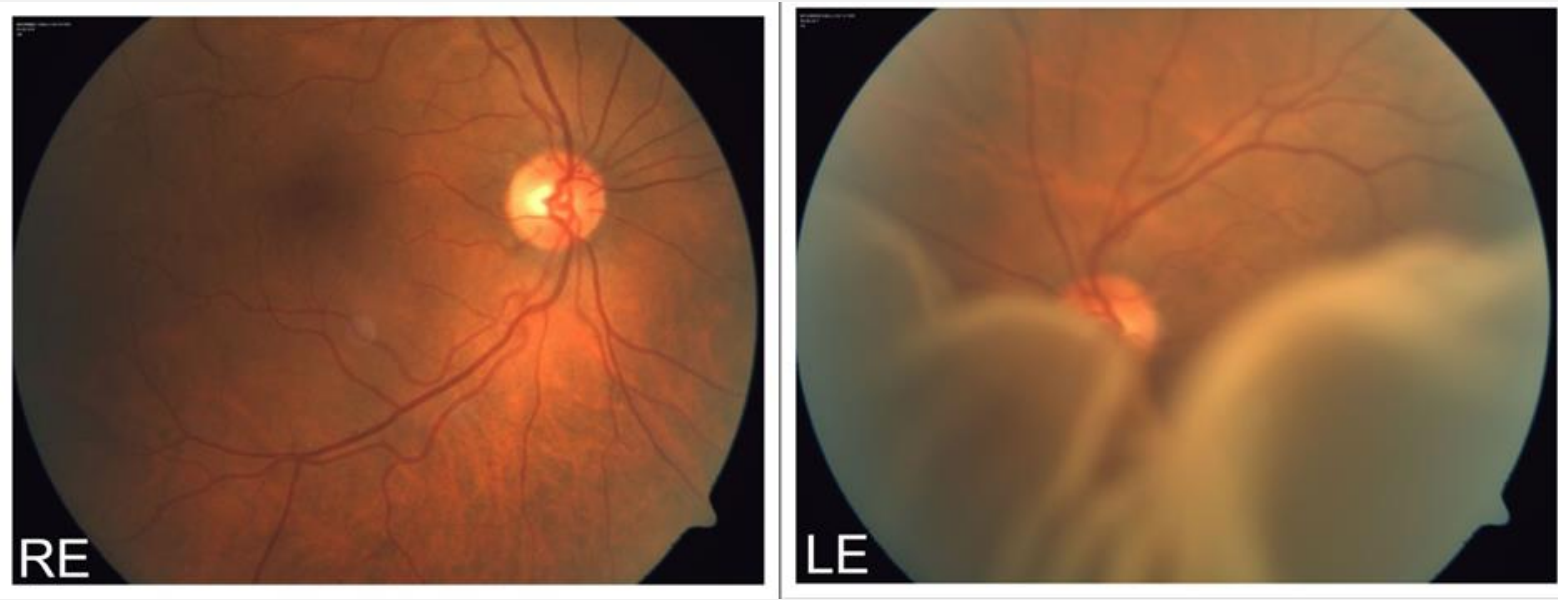

Figure 1: Fundus photograph of RE showing Mild NPDR and LE showing extensive exudative retinal detachment.
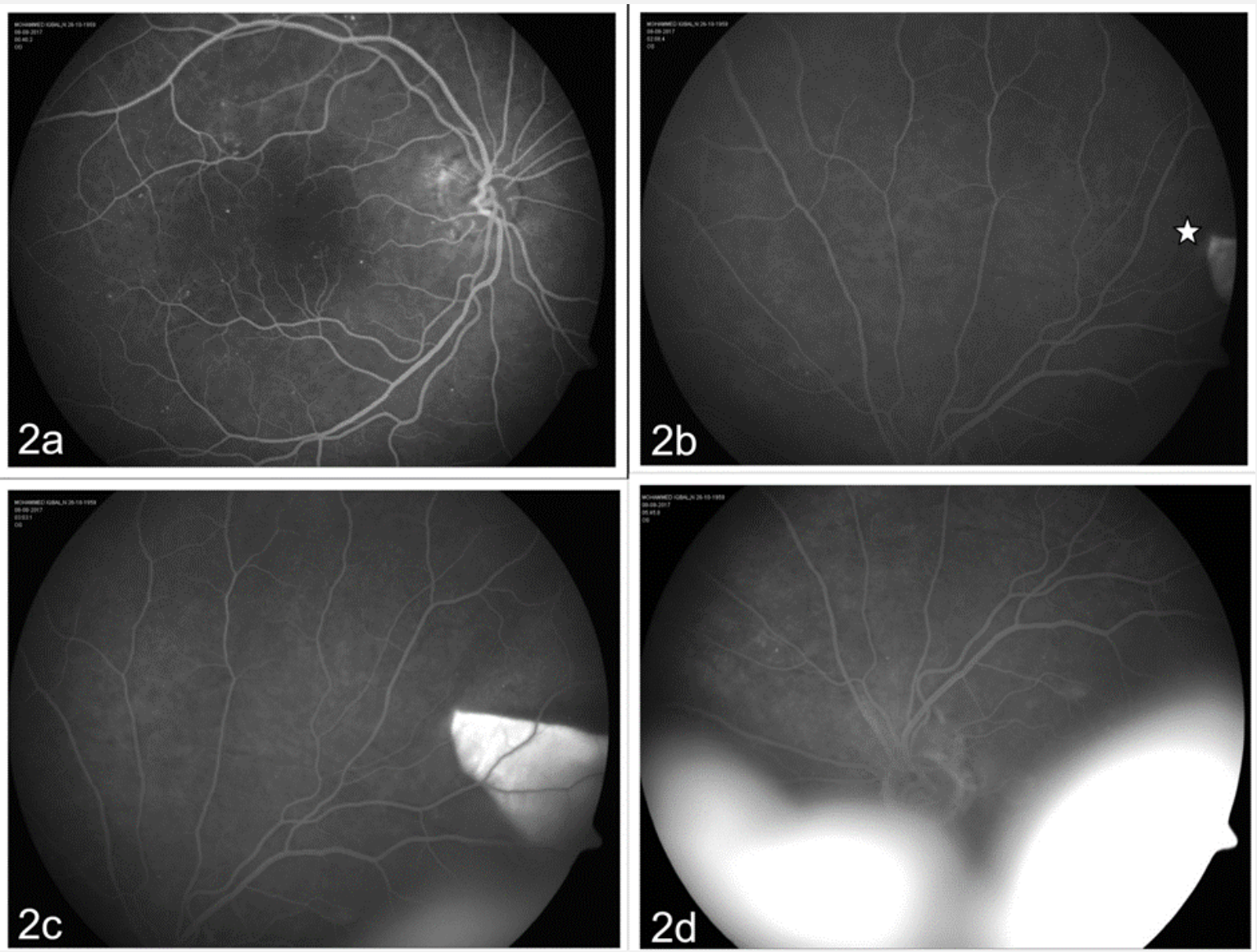

Figure 2: Fundus Fluorescein Angiogram. (2a) RE showing a few microaneurysms in the posterior pole s/o Mild NPDR. (2b) and (2c) LE showing transmitted choroidal hyper fluorsence (star) in the temporal mid periphery s/o RPE Rip. (2d) LE shows pooling of the dye in the exudative retinal detachment.

Shah VJ | Volume 2; Issue 2 (2021) | JOAR-2(2)-017 | Case Report

Citation: Shah VJ, et al. Spontaneous Giant Retinal Pigment Epithelium Rip with Exudative Retinal Detachment-An Unusual Presentation. J Ophthalmol Adv Res. 2021;2(2):1-6. 


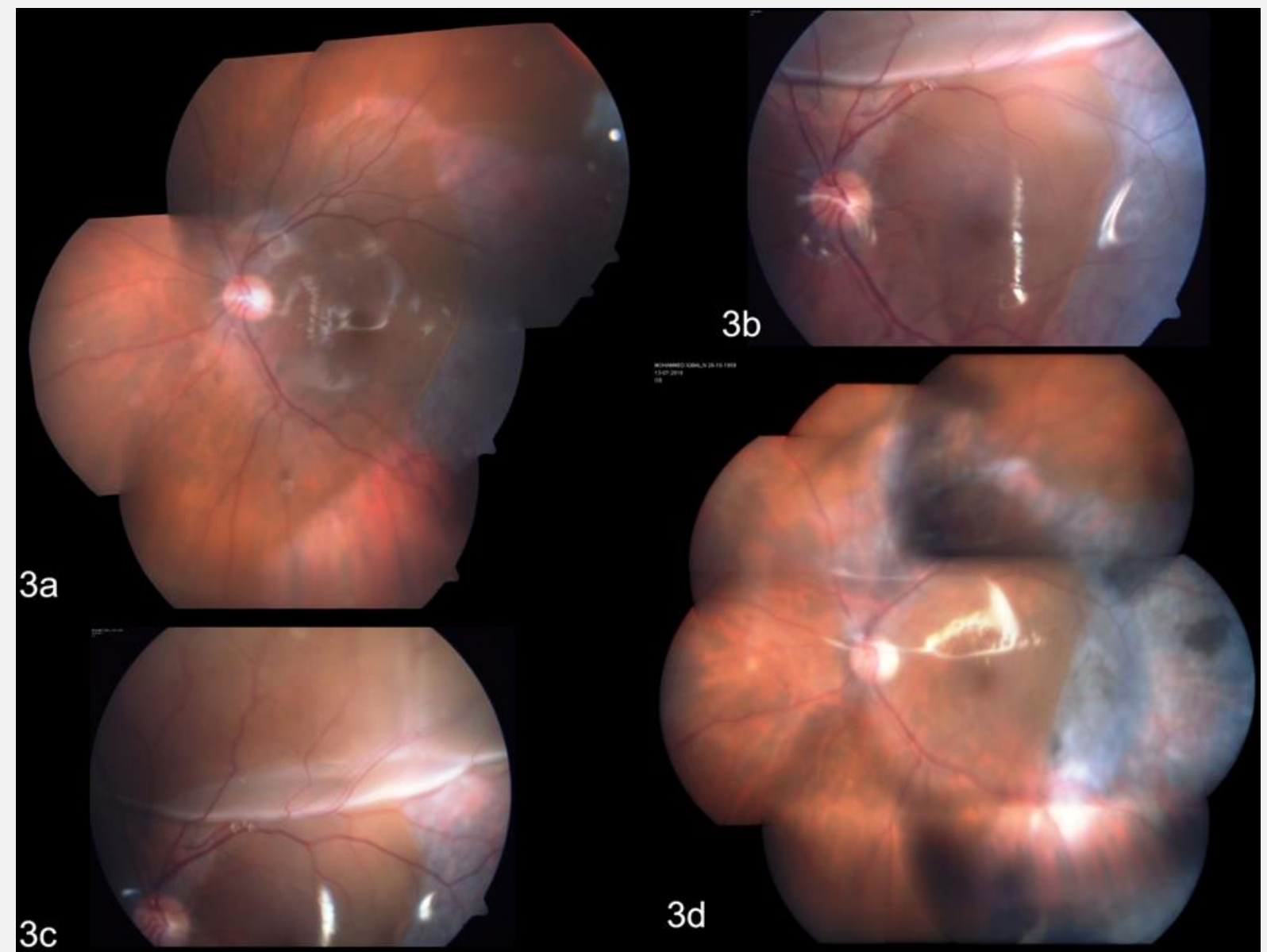

Figure 3: (3a) Colour Montage photo of the LE at 1st postoperative day showing attached retina under silicone oil. ( $3 \mathrm{~b}$ and $3 \mathrm{c}$ ) 1 week post-op fundus showing sub-retinal fluid in the superior quadrant along the extended RPE tear. (3d) Colour montage of LE showing attached retina under silicone oil at 6 months post-surgery.

\section{Discussion}

Tears in the RPE were first described in 1981 by Hoskins et al., [4]. RPE tears have increasingly been reported as a complication after Intravitreal anti-VEGF therapy in patients with PED due to exudative AMD [5]. Gass, postulated that leakage from an occult CNVM could cause serous PED, and progression of the leakage could result in hydrostatic pressure high enough to tear the RPE [6]. After anti-VEGF therapy, contraction of a CNVM adherent to the RPE increases causing rupture of the PED edge on the opposite side of CNVM, and the contractile force leads to rolling up of torn RPE layer next to CNVM [5]. Gass, also suggested that the thermal effect of laser photocoagulation might lead to CNVM contraction, inducing RPE tears [6].

The pathogenesis of RPE rip in cases other than wet AMD is not exactly known. It is said to be associated with choroidal vascular hyperpermeability which overwhelms the RPE initially, leading to serous RPE, and neurosensory detachments. The sub-RPE fluid causes tangential Shah VJ | Volume 2; Issue 2 (2021) | JOAR-2(2)-017 | Case Report

Citation: Shah VJ, et al. Spontaneous Giant Retinal Pigment Epithelium Rip with Exudative Retinal Detachment-An Unusual Presentation. J Ophthalmol Adv Res. 2021;2(2):1-6. 
stress, leading to formation of a tear. It has been postulated that the associated exudative detachment results from a loss of the barrier function of RPE [7]. Similarly, in our patient we postulate that the stress related to inguinal hernia surgery could have precipitated choroidal hyper permeability leading to formation of spontaneous RPE rip.

Sinawat S, et al., also reported a case of bilateral spontaneous RPE rip where no chorioretinal lesions were found, and they hypothesized that increased surface tension of RPE detachment is the etiology of RPE rips in their patient [8].

Healing of the RPE tears and resolution of Subretinal Fluid (SRF) can occur by many different mechanisms. It can be due to new hypopigmented RPE cells covering the area, or fibrous plaque developing in the bed of the tear as in most cases, and in few patients, the defect could remain unchanged or could be covered with normal-looking pigmented epithelium. [1,9] Considering the repair mechanisms of RPE rip, the underlying disease should be treated as early as possible.

The size of RPE tear, its location, and the viability of photoreceptors are the most important prognostic factors for visual outcome. Visual acuity is usually good with vascularised PED in the acute phase of a tear but is not sustained without treatment. Visual acuity can stabilize or improve with additional anti-VEGF injections. Studies observing the long-term visual outcome of patients with a tear during anti-VEGF therapy have revealed a poorer visual outcome in cases with subfoveal location and disciform scar. [1]

Hirota K, et al., reported management of extensive bullous exudative RD with Pars Plana vitrectomy and scleral window [10]. Doi M, et al., treated their patient of RPE tear post blunt trauma with cryotherapy but the exudative detachment increased for which they performed sclerectomy and sclerostomy [2]. Sunny CLA, Callie KLK reported a case of spontaneous RPE rip under the fovea with preserved good visual acuity for one year without intervention. However, the reason for photoreceptor preservation post RPE rip was not known in their case and they suggested further research is needed in RPE repair mechanisms for deciding whether surgical intervention or Anti-VEGF are required, or such patients can be observed [11].

In our case, vitrectomy was advised as there was a significant vision drop within one week despite medical management of exudative retinal detachment. Prolonged large bullous retinal detachment can cause permanent RPE damage, in our case despite early intervention the RPE degeneration at macula lead to suboptimal visual recovery. During vitrectomy, there was an extension of the RPE tear superiorly along the arcade up to the disc and due to this reason silicone oil was preferred as tamponade over gas, and at 1 week follow-up SRF developed in the superior quadrant along the extended RPE tear. This can be explained due to the compromised RPE pumping function in the area leading to fluid accumulation. The patient was observed as it has been reported that the neighboring RPE cells enlarge and healing occurs which helps in removal of fluid [1]. Also Silicone oil provides a longer tamponade effect which helped in reducing the extent of detachment till the SRF got absorbed. Over the next follow-

Shah VJ | Volume 2; Issue 2 (2021) | JOAR-2(2)-017 | Case Report

Citation: Shah VJ, et al. Spontaneous Giant Retinal Pigment Epithelium Rip with Exudative Retinal

Detachment-An Unusual Presentation. J Ophthalmol Adv Res. 2021;2(2):1-6.

DOI: http://dx.doi.org/10.46889/JOAR.2021.2205 
up, the retina was settled and SRF was absorbed completely which was maintained over 6 months.

\section{Conclusion}

Thus we report a case of exudative retinal detachment for which unexpectedly giant RPE tear was the cause without any obvious retinochoroidal lesions. Early surgical management in such cases can yield good visual outcomes, and extension of the RPE tear as a complication should be kept in mind while surgically managing such cases. The SRF usually resolves slowly and does not require immediate additional intervention.

\section{References}

1. Ersoz MG, Karacorlu M, Arf S, Muslubas IS, Hocaoglu M. Retinal pigment epithelium tears: classification, pathogenesis, predictors, and management. Survey Ophthalmol. 2017;62(4):493-505.

2. Doi M, Osawa S, Sasoh M, Uji Y. Retinal pigment epithelial tear and extensive exudative retinal detachment following blunt trauma. Graefes Arch Clin Exp Ophthalmol. 2000;238(7):621-4.

3. Casswell AG, Kohen D, Bird AC: Retinal pigment epithelial detachments in the elderly: classification and outcome. Br J Ophthalmol. 1985;69:397-403.

4. Hoskins A, Bird AC, Sehmi K. Tears of detached retinal pigment epithelium. Br J Ophthalmol. 1981;65:41722.

5. Nagiel A, Freund KB, Spaide RF, Munch IC, Larsen M, Sarraf D. Mechanism of retinal pigment epithelium tear formation following intravitreal anti-vascular endothelial growth factor therapy revealed by spectraldomain optical coherence tomography. Am J Ophthalmol. 2013;156(5):981e8.

6. Gass JD. Pathogenesis of tears of the retinal pigment epithelium. Br J Ophthalmol. 1984;68(8):513e9.

7. Ishida Y, Kato T, Minamoto A, Yokoyama T, Jian K, Mishima HK. Retinal pigment epithelial tear in a patient with central serous chorioretinopathy treated with corticosteroids. Retina. 2004;24:633-6.

8. Sinawat S, Bhoomibunchoo C, Yospaiboon Y, Sinawat S. Spontaneous bilateral retinal pigment epithelium rips with good visual acuity. J Med Assoc Thai. 2014;97(10):S115-9.

9. Laidlaw DA, Poynter R. Giant pigment epithelial tear and exudative retinal detachment complicating choroidal effusions. Am J Ophthalmol. 1998;126(3):448-50.

10. Hirota K, Hirakata A, Inoue M, Hiraoka T. Bilateral exudative retinal detachment due to retinal pigment epithelial tears successfully treated by vitrectomy and scleral window surgery. Acta Ophthalmol. 2012;90:e325-6.

11. Sunny CLA, Callie KLK. Spontaneous retinal pigment epithelium rips under fovea with preserved good visual acuity outcome. J Healthcare. 2020;3(1):33-7.

Shah VJ | Volume 2; Issue 2 (2021) | JOAR-2(2)-017 | Case Report 